\author{
М.Н. РЫБАКОВ
}

\title{
Неразрешимость модальных логик одноместного предиката ${ }^{1}$
}

\author{
Рыбаков Михаил Николаевич \\ Математический факультет, \\ Тверской государственный университет. \\ Российская федерация, 170100, г. Тверь, ул. Желябова, 33. \\ E-mail: m_rybakov@mail.ru
}

Рассматриваются модальные предикатные логики в языке, содержащем только одноместные предикатные буквы. Показано, что любая логика, содержащаяся в QS5, QGLLin или QGrz.3 является алгоритмически неразрешимой в языке с одной одноместной предикатной буквой (как при наличии, так и при отсутствии в логике формулы Баркан). Также показано, что логики конечных шкал Крипке (как с расширяющимися, так и с постоянными областями) для QK, QT, QD, QK4, QS4, QS5, QGL, QGrz и многих других не являются рекурсивно перечислимыми в языке с одной одноместной предикатной буквой. Тем не менее табличные логики и логики шкал Крипке с ограничением на число миров, достижимых из произвольного мира, разрешимы в языке с бесконечным множеством одноместных предикатных букв.

Ключевые слова: модальная логика, логика первого порядка, разрешимость

\section{1. Введение}

А. Чёрч и А. Тьюринг доказали, что логика предикатов QCL не является алгоритмически разрешимой $[8,9,16,17]$, т. е. не существует такой эффективной процедуры, которая по произвольной формуле логики предикатов выясняет, является ли эта формула тождественно истинной. Тем не менее QCL содержит довольно выразительные разрешимые фрагменты. Например, разрешимым является монадический фрагмент QCL, т. е. фрагмент QCL, образованный формулами в языке, содержащем только одноместные предикатные буквы. Действительно, с помощью одноместных предикатных букв $P_{1}, \ldots, P_{n}$ в

\footnotetext{
${ }^{1}$ Работа выполнена при поддержке РФФИ, проекты 16-07-01272 и 17-03-00818.
}

(c) Рыбаков М.H. 
модели можно различить не более чем $2^{n}$ элементов, а значит, чтобы выяснить, является ли тождественно истинной формула, содержащая в качестве предикатных букв только $P_{1}, \ldots, P_{n}$, достаточно проверить, истинна ли она в моделях, число элементов которых не превышает $2^{n}$. Незначительная модификация этой идеи позволяет получить разрешающие процедуры для монадического фрагмента QCL с равенством, см. [1], глава 25; более того, доказана даже разрешимость монадических фрагментов QCL, в которых дополнительно используются и бинарные буквы, но с определенными ограничениями [14].

Для неклассических логик предикатов ситуация с разрешимостью их монадических фрагментов выглядит совершенно иначе. С.Крипке показал $[13]^{2}$, что формула вида $R(x, y)$ может быть промоделирована в QS5 формулой вида $\diamond(P(x) \wedge Q(y))$. С учетом того, что фрагмент QCL в языке с одной бинарной предикатной буквой алгоритмически неразрешим (см., например, [1], глава 25), мы сразу получаем, что фрагмент QS5 в языке с двумя одноместными предикатными буквами также алгоритмически неразрешим; более того, этот результат автоматически распространяется на любую модальную предикатную логику, допускающую шкалу Крипке, содержащую мир, множество достижимых миров из которого бесконечно. Чуть позже С.Ю.Маслов, Г.Е. Минц и В.П. Оревков показали, что для неразрешимости интуиционистской логики предикатов вообще достаточно использовать одну одноместную предикатную букву [3], откуда следует неразрешимость QS4 в языке с одной одноместной предикатной буквой (см. также $[4,10])$. Отметим также, что во введении работы [11] утверждается неразрешимость логики QS5 в языке с одной одноместной предикатной буквой, но в [11] этот результат приводится без каких-либо пояснений.

Мы покажем, что неразрешимыми являются «почти все» модальные логики предикатов: для неразрешимости достаточно одной одноместной буквы в языке и отсутствия в логике формул, ограничивающих ширину ветвления в ее шкалах (при этом полнота по Крип-

\footnotetext{
${ }^{2}$ Русский перевод этой статьи можно найти в [6].
} 
ке не требуется). Во многих логиках формулу $\diamond(P(x) \wedge Q(y))$ можно промоделировать формулой $\diamond(P(x) \wedge \diamond P(y))$ : для этого достаточно, чтобы логика допускала иррефлексивные деревья высоты 2, в которых из корня достижимо бесконечно много миров. Этому условию удовлетворяют, например, все логики, содержащиеся в QGL. Ho, скажем, в случае QS5 сталкиваемся с затруднением: в QS5 формула $\diamond(P(x) \wedge \diamond P(y))$ эквивалентна формуле $\diamond P(x) \wedge \diamond P(y)$, и соответствующее ей бинарное отношение $R$ обладает некоторыми специфическими свойствами (например, из истинности $R(x, y)$ следует, что истинны также $R(y, x), R(x, x)$ и $R(y, y))$. Конечно, если в языке имеются две $\mathbf{S 5}$-модальности, то $R(x, y)$ моделируется, например, формулой $\diamond_{1}\left(P(x) \wedge\left(\neg P(y) \rightarrow \diamond_{2} P(y)\right) \wedge\left(P(y) \rightarrow \diamond_{2} \neg P(y)\right)\right)$. Ниже будет показано, как обойти указанное затрудение, используя лишь одну модальность.

\section{2. Синтаксис и семантика}

Будем считать, что модальный предикатный язык содержит счетное множество предметных переменных, счетное множество предикатных букв (для любой местности), символы связок $\wedge, \vee, \rightarrow, \neg$, логическую константу $\perp$, модальность $\square$, кванторный символ $\forall$, скобки и запятую. Формулы строятся обычным образом из элементарных формул вида $\perp$ и $P\left(x_{1}, \ldots, x_{n}\right)$, где $P-n$-местная предикатная буква, а $x_{1}, \ldots, x_{n}$ - предметные переменные, с помомощью связок $\wedge$, $\vee, \rightarrow, \neg$, модальности $\square$ и кванторов вида $\forall x$, где $x-$ предметная переменная.

Шкалой Крипке называем набор $\mathfrak{F}=\langle W, R\rangle$, где $W$ - непустое множество, $R$ - бинарное отношение на $W$. Элементы $W$ называем мирами, а отношение $R$ - отношением достижимости.

Шкалой Крипке с предметной областью (или предикатной шкалой Крипке) называем набор $\mathfrak{F}(D)=\langle W, R, D\rangle$, где $\langle W, R\rangle$ - шкала Крипке, а $D-$ функция, которая каждому миру $w \in W$ сопоставляет непустое множество $D(w)$, причем функция $D$ удовлетворяет следующему условию: если $w R w^{\prime}$, то $D(w) \subseteq D\left(w^{\prime}\right)$. Множество $D(w)$ называем предметной областью мира $w$. 
Шкалу $\mathfrak{F}(D)=\langle W, R, D\rangle$ называем шкалой с постоянными областями, если из $w R w^{\prime}$ следует, что $D(w)=D\left(w^{\prime}\right)$.

Моделью Крипке называем набор $\mathfrak{M}=\langle W, R, D, I\rangle$, где $\langle W, R, D\rangle$ - шкала с предметной областью, а $I-$ интерпретация предикатных букв в мирах множества $w$, т. е. функция, сопоставляющая каждому миру $w \in W$ и каждой $n$-местной предикатной букве $P$ некоторое $n$-местное отношение $I(w, P)$ на множестве $D(w)$.

Интерпретация предметных переменных - это функция $g$, которая каждой предметной переменной $x$ сопоставляет некоторый элемент $g(x)$. Содержательно $g(x)$ можно мыслить как элемент предметной области некоторого мира модели, но, вообще говоря, для дальнейших формальных определений это не имеет принципиального значения.

Определим рекурсивно отношение истинности формулы $\varphi$ в миpe $w$ модели $\mathfrak{M}=\langle W, R, D, I\rangle$ при интерпретации $g$. Если это отношение выполнено, то пишем $\mathfrak{M}, w \models^{g} \varphi$. Итак:

- $\mathfrak{M}, w \forall^{g} \perp$;

- $\mathfrak{M},\left.w\right|^{g} P\left(x_{1}, \ldots, x_{n}\right)$, если $\left\langle g\left(x_{1}\right), \ldots, g\left(x_{n}\right)\right\rangle \in I(w, P)$;

- $\mathfrak{M}, w \models^{g} \varphi^{\prime} \wedge \varphi^{\prime \prime}$, если $\mathfrak{M}, w \models^{g} \varphi^{\prime}$ и $\mathfrak{M}, w \models^{g} \varphi^{\prime \prime}$;

- $\mathfrak{M}, w \models^{g} \varphi^{\prime} \vee \varphi^{\prime \prime}$, если $\mathfrak{M}, w \models^{g} \varphi^{\prime}$ или $\mathfrak{M}, w \models^{g} \varphi^{\prime \prime}$;

- $\mathfrak{M}, w=^{g} \varphi^{\prime} \rightarrow \varphi^{\prime \prime}$, если $\mathfrak{M}, w \not^{g} \varphi^{\prime}$ или $\mathfrak{M}, w \models^{g} \varphi^{\prime \prime}$;

- $\mathfrak{M}, w=^{g} \neg \varphi^{\prime}$, если $\mathfrak{M},\left.w \not\right|^{g} \varphi^{\prime}$;

- $\mathfrak{M}, w=^{g} \square \varphi^{\prime}$, если для любого $w^{\prime} \in W$ из условия $w R w^{\prime}$ следует, что $\mathfrak{M}, w^{\prime} \models^{g} \varphi^{\prime}$;

- $\mathfrak{M}, w \models^{g} \forall x \varphi^{\prime}$, если для любой интерпретации $g^{\prime}$, отличающейся от $g$ только, быть может, значением на $x$, и такой, что $g^{\prime}(x) \in D(w)$, выполняется $\mathfrak{M}, w \models g^{\prime} \varphi^{\prime}$.

Формулу $\varphi$ считаем истинной в мире $w$ модели $\mathfrak{M}$, если $\varphi$ истинна в $w$ при любой интерпретации предметных переменных, которая 
интерпретирует каждую свободную переменную формулы $\varphi$ элементом из $D(w)$; в этом случае пишем $\mathfrak{M}, w \models \varphi$. Формулу $\varphi$ считаем истинной в модели $\mathfrak{M}$, если $\mathfrak{M}, w \models \varphi$ для каждого мира $w$ модели $\mathfrak{M}$; в этом случае пишем $\mathfrak{M}=\varphi$. Формулу $\varphi$ считаем истинной в шкале $\mathfrak{F}(D)$, если $\varphi$ истинна в каждой модели, определенной на $\mathfrak{F}(D)$; в этом случае пишем $\mathfrak{F}(D) \models \varphi$. Формулу $\varphi$ считаем истинной в шкале $\mathfrak{F}$, если $\varphi$ истинна в каждой шкале вида $\mathfrak{F}(D)$; в этом случае пишем $\mathfrak{F}=\varphi$. Наконец, формулу $\varphi$ считаем истинной в классе шкал, если она истинна в каждой шкале из этого класса.

Если $\varphi\left(x_{1} \ldots, x_{n}\right)$ - формула со свободными переменными $x_{1}, \ldots, x_{n}$ и $a_{1}, \ldots, a_{n}$ - элементы предметной области некоторого мира некоторой модели, то в этом случае иногда будем использовать запись $\varphi\left[a_{1}, \ldots, a_{n}\right]$, понимая ее так, что переменные $x_{1}, \ldots, x_{n}$ интерпретируются элементами $a_{1}, \ldots, a_{n}$, соответственно.

\section{3. Несколько вспомогательных фактов}

Для доказательства неразрешимости QS5 в языке с одной одноместной предикатной буквой нам понадобится несколько известных фактов, касающихся неразрешимости некоторых теорий бинарных отношений.

Пусть SIB - теория симметричного иррефлексивного бинарного отношения, т. е. множество всех первопорядковых формул в языке с одной бинарной предикатной буквой, истинных в моделях, где эта буква интерпретируется симметричным иррефлексивным отношением.

ФАКТ 1. Теория SIB алгоритмически неразрешима.

ДокАзАТЕЛЬСтво. В [1] в главе 25 приводится доказательство неразрешимости логики предикатов в языке с одной двухместной предикатной буквой. В [12] в $\$ 10$ показано, как бинарные отношения можно промоделировать симметричными иррефлексивными отношениями (см.также [15]). Как следствие получаем неразрешимость SIB. 
Пусть $s i b=\forall x \forall y(P(x, y) \rightarrow P(y, x)) \wedge \forall x \neg P(x, x)$. Истинность формулы $s i b$ в модели означает, что букве $P$ в этой модели сопоставлено симметричное иррефлексивное бинарное отношение.

ЗАМЕЧАНИЕ 1. $\varphi \in \mathbf{S I B} \Longleftrightarrow s i b \rightarrow \varphi \in \mathbf{Q C L}$.

Пусть FSIB - теория конечных моделей теории SIB.

ФАКТ 2. Теория FSIB не является рекурсивно перечислимой.

ДоказАтельство. В силу теоремы М.А. Трахтенброта [5], теория конечных моделей не является рекурсивно перечислимой. Используя аргументацию из [1] и [12], получаем неперечислимость FSIB.

ЗАМЕЧАНИЕ 2. $\varphi \in$ FSIB $\Longleftrightarrow s i b \rightarrow \varphi \in$ FQCL.

\section{4. Погружение}

Для первопорядковой формулы $\psi$ в языке с одной бинарной предикатной буквой $P$ обозначим через $\psi^{*}$ модальную формулу, получающуюся из $\psi$ заменой каждого вхождения вида $P(x, y)$ вхождением $\square(\neg Q(x) \vee \neg Q(y))$.

УТВЕРЖДДЕНЕ 1. Если $\varphi-$ безмодалъная первопорядковая замкнутая формула в языке с одной бинарной предикатной буквой $P$, то

$$
\varphi \in \mathbf{S I B} \Longleftrightarrow(\operatorname{sib} \rightarrow \varphi)^{*} \in \mathbf{Q S 5}
$$

ДоказАтеЛьство. Пусть $\varphi \in \mathbf{S I B}$. Тогда $s i b \rightarrow \varphi \in \mathbf{Q C L}$, и значит, $s i b \rightarrow \varphi \in$ QS5. Логика QS5 замкнута по правилу подстановки, а $(s i b \rightarrow \varphi)^{*}$ получена из $s i b \rightarrow \varphi$ с помощью этого правила, следовательно, $(\text { sib } \rightarrow \varphi)^{*} \in \mathbf{Q S 5}$.

Пусть $\varphi \notin \mathbf{S I B}$. Тогда формула $\varphi$ опровергается в некоторой алгебре $\mathfrak{A}=\langle A, S\rangle$, где $A-$ непустая предметная область, $S$ - симметричное иррефлексивное бинарное отношение на $A$, соответствующее букве $P$.

Пусть $W=A \times A, R=W \times W, D(w)=A$ для каждого $w \in W$. Определим модель $\mathfrak{M}=\langle W, R, D, I\rangle$, положив для всяких $a, b, c \in A$

$$
\mathfrak{M},\langle a, b\rangle \models Q[c] \leftrightharpoons c \in\{a, b\} \text { и неверно, что } a S b .
$$


Тогда для любых $a, b \in A$

$$
\mathfrak{M} \models \square(\neg Q[a] \vee \neg Q[b]) \Longleftrightarrow a S b,
$$

из чего заключаем, что $\mathfrak{M} \not \models \varphi^{*}$. Осталось заметить, что $\mathfrak{M} \models s i b^{*}$, a значит, $\mathfrak{M} \not \models(s i b \rightarrow \varphi)^{*}$. Следовательно, $(\text { sib } \rightarrow \varphi)^{*} \notin \mathbf{Q S 5}$.

Извлечем следствия из доказанного утверждения.

СЛЕДСТВИЕ 1. Логика QS5 в языке с одной одноместной предикатной буквой не является алгоритмически разрешимой.

На самом деле справедливо более сильное утверждение.

СЛЕДСТВИЕ 2. Пусть $L-$ произвольное множество бормул в модальном языке первого порядка с одной одноместной предикатной буквой, содержащее в себе все подстановочные примерь формул из QCL u содержсащееся в QS5. Тогда L не является разрешимым.

ДокАзАтЕЛьство. Достаточно показать, что если $\varphi-$ безмодальная первопорядковая замкнутая формула в языке с одной бинарной предикатной буквой $P$, то

$$
\varphi \in \mathbf{S I B} \Longleftrightarrow(s i b \rightarrow \varphi)^{*} \in L .
$$

Если $\varphi \in \mathbf{S I B}$, то sib $\rightarrow \varphi \in \mathbf{Q C L}$, и тогда $(s i b \rightarrow \varphi)^{*} \in L$, так как все подстановочные примеры формул из QCL содержатся в $L$. Если же $\varphi \notin \mathbf{S I B}$, то, как было показано, $(s i b \rightarrow \varphi)^{*} \notin \mathbf{Q S 5}$, а значит, $(s i b \rightarrow \varphi)^{*} \notin L$, поскольку $L \subseteq \mathbf{Q S 5}$.

СЛЕДСТвиЕ 3. Логики QK, QK4, QT, QD, QS4 в язие с одной одноместной предикатной буквой не являются алгоритмически разрешимыми.

Заметим, что в доказательстве утверждения 1 можно считать, что $A$ - счетное множество (в силу теоремы Лёвенгейма-Сколема; см., например, [2], следствие 2.16), поэтому, по сути, важно лишь то, чтобы логика допускала шкалу, где имеется мир, из которого достижимо бесконечно много других миров. Другими словами, справедлива следующая «техническая» лемма. 
ЛЕмма 1. Пусть L - логика, допускающая шкалу Крипке, содержащую мир, из которого достижимо бесконечно много других миров. Тогда если $\varphi-$ безмодальная первопорядковая замкнутая формула в языке с одной бинарной предикатной буквой $P$, то

$$
\varphi \in \mathbf{S I B} \Longleftrightarrow(s i b \rightarrow \varphi)^{*} \in L
$$

Из этой леммы мы получаем, что предикатные модальные логики, не имеющие ограничений на ширину ветвления, неразрешимы даже в языке с одной одноместной предикатной буквой; то же касается и логик, допускающих транзитивные шкалы с бесконечными цепями миров, видимыми из некоторого мира. Пусть $b d_{2}-$ формула, ограничивающая высоту шкалы Крипке числом 2 (так, можно взять $b d_{2}=\diamond\left(\square p_{2} \wedge \neg\left(\diamond \square p_{1} \rightarrow p_{1}\right)\right) \rightarrow p_{2}$, где $p_{1}$ и $p_{2}$ - пропозициональные переменные; см., например, [7, с. 81]).

СЛЕДСТВИЕ 4. Логики QGL $\oplus b d_{2}$, QGrz $\oplus b d_{2}$, QGLLin, QGrz.3 в языке с одной одноместной предикатной буквой не являются алгоритмически разрешимыми.

Отметим также, что все результаты останутся справедливыми, если к рассмотренным выше логикам добавить формулу Баркан $b f=\forall x \square Q(x) \rightarrow \square \forall x Q(x)$, требующую постоянства предметных областей: эта формула принадлежит логике QS5 и не мешает описанной конструкции.

Подведем итог.

ТЕОРема 1. Пусть логика $L$ содержит QCL и содержится в одной из логик QS5, QGL $\oplus b d_{2} \oplus b f, \mathbf{Q G r z} \oplus b d_{2} \oplus b f$, QGLLin $\oplus b f$, QGrz.3 $\oplus b f$. Тогда фрагмент $L$ с одной одноместной предикатной буквой не являются алгоритмически разрешимыми.

Теперь рассмотрим несколько случаев, когда условия леммы 1 не выполнены. 


\section{5. Табличные логики}

Пусть $L$ - модальная предикатная логика. По аналогии с пропозициональным случаем будем называть $L$ табличной, если $L$ совпадает со множеством формул, истинных в некоторой конечной шкале Крипке.

Пусть $L-$ табличная модальная предикатная логика и пусть $\mathfrak{F}=\langle W, R\rangle-$ конечная шкала, относительно которой полна $L$. Обозначим элементы множества $W$ через $w_{1}, \ldots, w_{m}$. Пусть $\varphi-$ формула, содержащая лишь одноместные предикатные буквы $P_{1}, \ldots, P_{n}$.

Лемма 2. Пусть $\mathfrak{F}(D) \not \models \varphi$. Тогда существует такая шкала $\mathfrak{F}\left(D^{\prime}\right)$, что $\mathfrak{F}\left(D^{\prime}\right)|\models \varphi,| D^{\prime}\left(w_{1}\right) \cup \ldots \cup D^{\prime}\left(w_{m}\right) \mid \leqslant 2^{m(n+1)}$, причем если $\mathfrak{F}(D)-$ шкала с постоянными областями, то $\mathfrak{F}\left(D^{\prime}\right)$ - тоже шкала с постоянными областями.

ДоказАтЕЛЬСтво. Пусть $\mathfrak{M}=\langle W, R, D, I\rangle$ - модель на предикатной шкале $\mathfrak{F}(D)$, в некотором мире $w_{k}$ которой опровергается формула $\varphi$. Пусть $\mathfrak{D}=D\left(w_{1}\right) \cup \ldots \cup D\left(w_{m}\right)$. Введем на множестве $\mathfrak{D}$ отношение эквивалентности $\sim$, положив $a \sim b$, если для каждого $i \in\{1, \ldots, m\}$ и каждого $j \in\{1, \ldots, n\}$ выполнены следующие условия:

(1) $a \in D\left(w_{i}\right) \Longleftrightarrow b \in D\left(w_{i}\right)$;

(2) $\mathfrak{M}, w_{i} \models P_{j}[a] \Longleftrightarrow \mathfrak{M}, w_{i} \models P_{j}[b]$.

Пусть $\bar{a}=\{c \in \mathfrak{D}: c \sim a\}$. Пусть также для каждого $w_{i} \in W$

$$
D^{\prime}\left(w_{i}\right)=D\left(w_{i}\right) / \sim=\left\{\bar{a}: a \in D\left(w_{i}\right)\right\} .
$$

Поскольку из условия $D\left(w_{i}\right) \subseteq D\left(w_{j}\right)$ следует, что $D^{\prime}\left(w_{i}\right) \subseteq D^{\prime}\left(w_{j}\right)$, получаем, что $\mathfrak{F}\left(D^{\prime}\right)$ - шкала Крипке. Определим на $\mathfrak{F}\left(D^{\prime}\right)$ модель $\mathfrak{M}^{\prime}=\left\langle W, R, D^{\prime}, I^{\prime}\right\rangle$, положив

$$
I^{\prime}\left(w_{i}, P_{j}\right)=\left\{\langle\bar{a}\rangle:\langle a\rangle \in I\left(w_{i}, P_{j}\right)\right\} .
$$

Другими словами,

$$
\mathfrak{M}^{\prime}, w_{i} \models P_{j}[\bar{a}] \Longleftrightarrow \mathfrak{M}, w_{i} \models P_{j}[a] .
$$


Последняя эквивалентность несложно распространяется и на другие формулы. Если $\psi\left(x_{1}, \ldots, x_{s}\right)$ - подформула формулы $\varphi$ со свободными переменными $x_{1}, \ldots, x_{s}$, то

$$
\mathfrak{M}^{\prime}, w_{i} \models \psi\left[\bar{a}_{1}, \ldots, \bar{a}_{s}\right] \Longleftrightarrow \mathfrak{M}, w_{i} \models \psi\left[a_{1}, \ldots, a_{s}\right]
$$

В частности, получаем, что $\mathfrak{M}^{\prime}, w_{k} \not \neq \varphi$.

Осталось заметить, что эквивалентность, определяемая лишь условием (1), разбивает $\mathfrak{D}$ на не более чем $2^{m}$ классов, при этом каждый такой класс разбивается эквивалентностью, определяемой лишь условием (2), на не более чем $2^{m n}$ классов. Следовательно, $\left|D^{\prime}\left(w_{1}\right) \cup \ldots \cup D^{\prime}\left(w_{n}\right)\right| \leqslant 2^{m(n+1)}$. Кроме того, если $\mathfrak{F}(D)-$ шкала с постоянными областями, то $\mathfrak{F}\left(D^{\prime}\right)$ - тоже шкала с постоянными областями.

УТВЕРЖДЕНИЕ 2. Унарный фрагмент табличной логики алгоритмически разрешим.

ДокАЗАтельство. Пусть $L-$ табличная логика. Тогда существует конечная шкала $\mathfrak{F}$, относительно которой полна $L$. Пусть $m-$ число миров в $\mathfrak{F}$. Пусть $\varphi$ - произвольная формула из унарного фрагмента, $n$ - число одноместных предикатных букв в $\varphi$. Рассмотрим все модели на шкале $\mathfrak{F}$, число элементов в предметных областях которых не превосходит $2^{m(n+1)}$. Эти модели образуют конечное множество (если их рассматривать с точностью до изоморфизма, конечно). Для каждой из этих моделей проверим, истинна ли в ней формула $\varphi$. Если нет, то $\varphi \notin L$. Если да, то, согласно лемме 2, $\varphi \in L$.

ЗАмечАНИЕ 3. Унарный фрагмент табличной логики, содержащей бормулу Баркан, алгоритмически разрешим.

Стоит также отметить, что алгоритмическая разрешимость монадического фрагмента сохранится и для некоторых нетабличных логик. Например, она сохранится для логик, определяемых шкалами, в которых из каждого мира достижимо не более чем $k$ миров, где $k$ - фиксированное число. Действительно, если тестируемая формула $\varphi$ содержит $n$ одноместных предикатных букв и имеет модаль- 
ную глубину $\operatorname{md}(\varphi)$, то для проверки истинности $\varphi$ в мире $w$ модели с ветвлением, ограниченным числом $k$, достаточно рассмотреть лишь часть этой модели, образованной мирами, достижимыми из $w$ не более чем за $\operatorname{md}(\varphi)$ шагов. $\mathrm{C}$ каждым шагом число новых миров возрастает не более чем в $k$ раз, поэтому общее количество интересующих нас миров не превосходит числа $m=1+k+k^{2}+\ldots+k^{\operatorname{md}(\varphi)}$, т. е. числа

$$
m=\frac{k^{\operatorname{md}(\varphi)+1}-1}{k-1} .
$$

В этом случае, используя аргументацию доказательства леммы 2 , мы получаем, что для выяснения принадлежности формулы $\varphi$ логике $L$, определяемой подобным классом шкал, достаточно рассматривать конечные шкалы глубины $\operatorname{md}(\varphi)$ с указанным числом миров, предметная область которых содержит не более чем $2^{m(n+1)}$ элементов.

Пусть $b a_{k}$ - формула, ограничивающая количество миров, достижимых из произвольного мира шкалы, числом $k$. Например, можно взять $b a_{k}=\square \alpha_{1} \vee \ldots \vee \square \alpha_{k+1}$, где $\alpha_{m}=p_{1} \wedge \ldots \wedge p_{m-1} \wedge \neg p_{m} \wedge p_{m+1} \wedge \ldots \wedge p_{k+1}\left(p_{1}, \ldots, p_{k+1}-\right.$ пропозициональные переменные).

СледСТвИЕ 5. Монадические фрагменты логик $\mathbf{Q K} \oplus b a_{k}, \mathbf{Q T} \oplus b a_{k}$, $\mathbf{Q D} \oplus b a_{k}, \mathbf{Q K B} \oplus b a_{k}, \mathbf{Q K} \oplus b a_{k} \oplus b f, \mathbf{Q T} \oplus b a_{k} \oplus b f, \mathbf{Q D} \oplus b a_{k} \oplus b f$, QKB $\oplus b a_{k} \oplus b f$ алгоритмически разрешимьь.

\section{6. Логики конечных моделей}

Ситуация в корне меняется, если число миров, достижимых из некоторого мира, может быть сколь угодно большим. Пусть $\mathfrak{C}-$ класс конечных шкал Крипке, в котором для каждого $n$ имеется шкала, из некоторого мира которой достижимо не менее $n$ миров. Пусть $L(\mathfrak{C})$ - логика этого класса, т. е. множество формул, истинных в каждой шкале из $\mathfrak{C}$.

В этом случае перевод, заменяющий подформулы вида $P(x, y)$ на $\square(\neg Q(x) \bigvee \neg Q(y))$, погружает рекурсивно неперечислимую теорию FSIB в $L(\mathfrak{C})$. 
УТВЕРЖДЕНИЕ 3. Если $\varphi-$ безмодалъная первопорядковая замкнутая формула в языке с одной бинарной предикатной буквой $P$, то

$$
\varphi \in \mathbf{F S I B} \Longleftrightarrow(s i b \rightarrow \varphi)^{*} \in L\left(\mathfrak{C}^{\mathfrak{r}}\right)
$$

ДоКАЗАТЕЛЬСтво. Пусть $\varphi \in$ FSIB. Для того, чтобы доказать, что $(s i b \rightarrow \varphi)^{*} \in L(\mathfrak{C})$, предположим, что это не так. Тогда в $\mathfrak{C}$ существует такая шкала $\mathfrak{F}=\langle W, R\rangle$, что для некоторой модели $\mathfrak{M}=\langle W, R, D, I\rangle$ и некоторого мира $w_{0} \in W$ имеем $\mathfrak{M}, w_{0} \not=(s i b \rightarrow \varphi)^{*}$. Пусть $m$ - число элементов в $W$. Согласно лемме 2 можно считать, что $D\left(w_{0}\right)$ содержит не более чем $2^{2 m}$ элементов (т.к. в нашем случае $n=1)$. Рассмотрим алгебру $\mathfrak{A}=\left\langle D\left(w_{0}\right), S\right\rangle$, где $S$ - это бинарное отношение, определенное следующим образом:

$$
a S b \leftrightharpoons \mathfrak{M}, w_{0} \models \square(\neg Q[a] \vee \neg Q[b])
$$

Тогда $\mathfrak{A}$ - конечная алгебра, в которой $S$ - симметричное иррефлексивное отношение (так как $\mathfrak{M}, w_{0}=s i b^{*}$ ), при этом если букву $P$ интерпретировать отношением $S$, то получим, что $\mathfrak{A} \forall \varphi$ (так как $\left.\mathfrak{M}, w_{0} \not \varphi^{*}\right)$. Получили противоречие с тем, что $\varphi \in$ FSIB. Значит, $(s i b \rightarrow \varphi)^{*} \in L(\mathfrak{C})$.

Пусть теперь $(s i b \rightarrow \varphi)^{*} \in L(\mathfrak{C})$. Предположим, что $\varphi \notin$ FSIB. В этом случае существует конечная алгебра $\mathfrak{A}=\langle A, S\rangle$, где $S-$ симметричное иррефлексивное бинарное отношение на $A$, в которой опровергается $\varphi$. Пусть $n=|A|$. В классе $\mathfrak{C}$ выберем шкалу $\mathfrak{F}=\langle W, R\rangle$, в которой существует мир $w_{0}$, из которого достижимо не менее чем $n^{2}$ миров, отличных от $w_{0}$. Во множестве миров, достижимых из $w_{0}$ и отличных от $w_{0}$, выберем подмножество из $n^{2}$ элементов, после чего его элементы проиндексируем двойными индексами из $A$; получим множество элементов вида $w_{a, b}$, где $a, b \in A$. Положим $D(w)=A$ для каждого $w \in W$. Определим модель $\mathfrak{M}=\langle W, R, D, I\rangle$, положив для всякого $w \in W$ и всякого $c \in A$

$$
\begin{aligned}
\mathfrak{M}, w \models Q[c] \leftrightharpoons & \text { существует такие } a, b \in A, \text { что } c \in\{a, b\}, \\
& w=w_{a, b} \text { и неверно, что } a S b .
\end{aligned}
$$


Тогда для любых $a, b \in A$

$$
\mathfrak{M}, w_{0} \models \square(\neg Q[a] \vee \neg Q[b]) \Longleftrightarrow a S b,
$$

из чего заключаем, что $\mathfrak{M}, w_{0} \not \models \varphi^{*}$. Теперь осталось заметить, что $\mathfrak{M}, w_{0} \models s i b^{*}$, а значит, $\mathfrak{M}, w_{0} \not=(s i b \rightarrow \varphi)^{*}$. Следовательно, $(s i b \rightarrow \varphi)^{*} \notin L(\mathfrak{C})$, что не так. Значит, $\varphi \in$ FSIB.

ЗАмечАнИЕ 4. Утверждение останется справедливым, если ограничиться шкалами с постоянными предметными областями.

Для модальной предикатной логики $L$ обозначим через $\mathbf{F} L$ логику конечных шкал логики $L$.

СлеДСтвиЕ 6. Логики FQK, FQT, FQK4, FQD, FQS4, FQS5, FQGL, FQGrz в языке с одной одноместной предикатной буквой не являются рекурсивно перечислимыми.

Поскольку во всех случаях используется один и тот же перевод, последнее утверждение можно усилить, распространив его на бесконечные классы логик. При этом учтем возможность ограничения на высоту шкал и на постоянство предметных областей.

ТЕОРЕма 2. Пусть $L-$ модальная предикатная логика, причем $\mathbf{F Q K} \subseteq L \subseteq \mathbf{F Q G L} \oplus b d_{2} \oplus b f, u л u \mathbf{F Q K} \subseteq L \subseteq \mathbf{F Q G r z} \oplus b d_{2} \oplus b f$, или FQK $\subseteq L \subseteq$ FQS5. Тогда фрагмент $L$ с одной одноместной предикатной буквой не являются рекурсивно перечислимым.

\section{Литература}

[1] Булос Дж., Джеффри Р. Вычислимость и логика. М.: Мир, 1994.

[2] Мендельсон Э. Введение в математическую логику. М.: Наука, 1984.

[3] Маслов С.Ю., Мини, Г.Е., Оревков В.П. Неразрешимость в конструктивном исчислении предикатов некоторых классов формул, содержащих только одноместные предикатные переменные // Докл. AH CCCP. T. 163. № 2. 1965. C. 295-297.

[4] Ръбаков M.Н. Об алгоритмической выразительности модального языка с одной лишь одноместной предикатной буквой // Логические исследования. Вып. 9. М.: Наука. 2002. С.179-201. 
[5] Трахтенброт Б.А. Невозможность алгорифма для проблемы разрешимости на конечных классах // Докл. АН СССР. Т. 70. № 4. 1950. C. 569-572.

[6] Фейс Р. Модальная логика. М.: Наука. 1974.

[7] Chagrov A.V., Zakharyaschev M.V. Modal Logic. Oxford University Press, 1997.

[8] Church A. An Unsolvable Problem of Elementary Number Theory // American Journal of Mathematics. 1936. Vol. 58. P. 345-363.

[9] Church A. A note on the Entscheidungsproblem // Journal of Symbolic Logic. 1936. Vol. 1. P. 40-41.

[10] Gabbay D.M. Semantical investigations in Heyting's intuitionistic logic // Synthese Library. Vol. 148. Reidel; Dordrecht: 1989.

[11] Gabbay D.M., Shehtman V.B. Undecidability of Modal and Intermediate First-Order Logics with Two Individual Variables // The Journal of Symbolic Logic. Sept. 1993. Vol. 58. № 3. P. 800-823.

[12] Kremer P. On the Complexity of Propositional Quantification in Intuitionistic Logic // The Journal of Symbolic Logic. 1997. Vol. 62. № 2. P. 529-544.

[13] Kripke S. The Undecidability of Monadic Modal Quantificational Theory // Zeitschrift für Mathematische Logik und Grundlagen der Mathematik. 1962. Vol. 8. P. 113-116.

[14] Motohashi N. A Decision Method for a Set of First Order Classical Formulas and its Applications to Decision Problem for Non-Classical Propositional Logics // J. Math. Soc. Japan. 1990. Vol. 42. № 1. P. $127-132$.

[15] Nerode A., Shore $R$. Second Order Logic and First Order Theories of Reducibility Orderings // The Kleene Symposium, J. Barwise, H. J. Keisler, K. Kuner, eds., North-Holland, Amsterdam. 1980. P. 181-200.

[16] Turing A.M. On Computable Numbers with an Application to the Entscheidungsproblem // Proc. London Maths. Soc. 1936. ser. 2. Vol. 42. P. 230-265.

[17] Turing A.M. On Computable Numbers, with an Application to the Entscheidungsproblem. A Correction // Proc. London Maths. Soc. 1937. ser. 2. Vol. 43. P. 544-546. 


\title{
M.N. RYBAKOV \\ Undecidability of Modal Logics of Unary Predicate $^{1}$
}

\author{
Rybakov Mikhail Nikolaevich \\ Mathematical Faculty, Tver State University. \\ 33 Zhelabova St., Tver, 170100, Russian Federation. \\ E-mail: m_rybakov@mail.ru
}

We consider first-order modal logics with unary predicate letters only. We show that any sublogic of QS5, QGLLin, or QGrz.3 is undecidable in the language with just one unary predicate letter (with or without Barcan formula). We also show that logics of finite Kripke models (with expanding or constant domains) for QK, QT, QD, QK4, QS4, QS5, QGL, QGrz, and some others are not recursively enumerable in the language with one unary letter. Nevertheless tabular logics and logics of Kripke frames with restrictions on the number of worlds accessible from any world are decidable in the language with infinitely many unary predicate letters.

Keywords: modal logic, first-order logic, decidability

\section{References}

[1] Boolos, G.S., Jeffrey, R.C. Computability and Logic. Third Edition. Cambrige University Press, 1989.

[2] Mendelson, E. Introduction to Mathematical Logic. D. van Nostrand Company, Inc., 1964.

[3] Maslov, S. Yu., Mints, G.E., Orevkov, V.P. "Nerazreshimost' v konstruktivnom ischislenii predicatov nekotoryh klassov formul, soderzhaschih tol'ko odnomestnye predikatnye peremennye" [Undecidability in constructive first-order calculus of some classes of formulas with only unary predicate letters], Doklady AN SSSR [Proceedings of AS USSR], 1965, vol. 163, № 2, pp. 295-297. (In Russian)

[4] Rybakov, M.N. "Ob algoritmicheskoy vyrasitel'nosti modal'nogo yazyka s odnoy lish' odnomestnoy predikatnoy bukvoy" [On algorithmic power of modal language with one unary predicate letter only], Logicheskie Issledovaniya [Logical Investigations], 2002, vol. 9. Moscow, Nauka, pp. 179-201. (In Russian)

\footnotetext{
${ }^{1}$ The paper is supported by Russian Foundation for Basic Research, projects №16-07-01272 and №17-03-00818.
} 
[5] Trakhtenbrot, B.A. "Nevozmozhnost' algoritma dlya problemy razreshimosti na konechnyh klassah" [Impossibility of an algorithm for decidability problem on finite classes], Doklady AN SSSR [Proceedings AS USSR], 1950, vol. 70, № 4, pp. 569-572. (In Russian)

[6] Feys, R. Modal'naya Logika [Modal Logic]. Moscow, Nauka, 1974. (In Russian).

[7] Chagrov, A.V., Zakharyaschev, M.V. Modal Logic. Oxford University Press, 1997.

[8] Church, A. "An Unsolvable Problem of Elementary Number Theory", American Journal of Mathematics, 1936, vol. 58, pp. 345-363.

[9] Church, A. "A note on the Entscheidungsproblem", Journal of Symbolic Logic, 1936, vol. 1, pp. 40-41.

[10] Gabbay, D.M. "Semantical investigations in Heyting's intuitionistic logic", Synthese Library, 1989, vol. 148, Reidel, Dordrecht.

[11] Gabbay, D.M., Shehtman, V.B. "Undecidability of Modal and Intermediate First-Order Logics with Two Individual Variables", The Journal of Symbolic Logic, Sept. 1993, vol. 58, № 3, pp.800-823.

[12] Kremer, P. "On the Complexity of Propositional Quantification in Intuitionistic Logic", The Journal of Symbolic Logic, 1997, vol. 62, № 2, pp. 529-544.

[13] Kripke, S. "The Undecidability of Monadic Modal Quantificational Theory", Zeitschrift für Mathematische Logik und Grundlagen der Mathematik, 1962, vol. 8, pp. 113-116.

[14] Motohashi, N. "A Decision Method for a Set of First Order Classical Formulas and its Applications to Decision Problem for Non-Classical Propositional Logics", J. Math.Soc.Japan, 1990, vol. 42, № 1, pp. 127-132.

[15] Nerode, A., Shore, R. "Second Order Logic and First Order Theories of Reducibility Orderings", The Kleene Symposium, J.Barwise, H.J.Keisler, K.Kuner, eds., North-Holland, Amsterdam, 1980, pp. 181-200.

[16] Turing, A.M. "On Computable Numbers with an Application to the Entscheidungsproblem", Proc. London Maths. Soc., 1936, ser. 2, vol. 42, pp. 230-265.

[17] Turing, A.M. "On Computable Numbers, with an Application to the Entscheidungsproblem. A Correction", Proc. London Maths. Soc., 1937, ser. 2, vol. 43 , pp. 544-546. 